\title{
CARACTERÍSTICAS FENOTÍPICAS DE PACIENTE CON CROMOSOMA 7 EN ANILLO
}

\author{
Carmen Rosa Dávila-Aliaga ${ }^{1}$, Stephanie Brigitte Del Castillo Bao², Rayza Guillén Zambrano ${ }^{3}$, \\ Lucena Angulo Ramirez ${ }^{4}$
}

\author{
INSTITUCIÓN \\ Departamento de Neonatología del Instituto Nacional Materno Perinatal.
}

\section{RESUMEN}

Se describe el caso de una lactante con microcefalia, retraso en el crecimiento pondoestatural y de lenguaje, nacida en el Instituto Materno Perinatal en quien se halló un cariotipo 46, XX, r(7) (p22q36) [25]. Los hallazgos fenotípicos observados en el propositus fueron compatibles con los pocos casos reportados en el mundo con cromosoma 7 en anillo.

Palabras clave: Cromosoma 7 en anillo; Microcefalia(Fuente: DeCS BIREME)

\section{PHENOTYTIC CHARACTERISTICS OF PATIENT WITH CHROMOSOME 7 IN RING}

\begin{abstract}
We describe the case of a female infant with microcephaly, delay in weight and height growth; speech and language, born in the Maternal Perinatal Institute with a karyotype 46, XX, r (7) (p22q36) [25]. The phenotypic findings observed in the propositus were compatible with the few cases reported in the world with ring chromosome 7 .
\end{abstract}

Key words: Ring chromosome 7; Microcephaly (Source: MeSH NLM).

\section{INTRODUCCIÓN}

Una célula diploide cuenta con dos copias de cromosomas, a diferencia de la monosomía, que es una alteración genética donde se cuenta con una única copia de estas, e incluso segmentos; dando lugar a enfermedades genéticas humanas ${ }^{1}$. Los cromosomas en anillo son una alteración estructural del material genético, en lugar de una pérdida o ganancia de éste, producto de una disfunción de los telómeros; y se atribuye a la muerte de células con aneuploidía secundaria, causada por inestabilidad durante la mitosis ${ }^{2}$; es común encontrarlo en solo una porción de todas las células analizadas, como mosaicismo $^{3}$, y suelen surgir de novo y no más del $1 \%$ son heredados ${ }^{2}$. Esta inestabilidad, nivel de mosaicismo y posición del punto de ruptura del cromosoma van a definir las características del paciente.

La monosomia 7 es una anomalía cromosómica poco frecuente, con una prevalencia mundial menor a 1/ 1000000 de casos $^{4}$. Con fenotipos variables, destacando el crecimiento (talla baja y microcefalia); además de la dismorfia facial (con microtia, fisuras palpebrales anómalas, ptosis palpebral, pliegues epicánticos, hiper-l hipotelorismo) y anomalías dermatológicas (nevus flammeus y pigmentados oscuros, manchas café con leche $)^{5}$.

La historia de la monosomia del cromosoma 7 inició en 1964 como un nuevo síndrome clínico, caracterizado por anemia refractaria con ausencia de uno de los cromosomas del grupo 6 - 12 en las células de la médula, que terminan con Leucemia mielomonocítica aguda ${ }^{6}$. Además, la evolución de la enfermedad también se ha asociado a mutaciones de la línea germinal GATA2 (3q21.3), neurofibromatosis, y neutropenia congénita severa.

\section{REPORTE DEL CASO}

Presentamos el caso de una lactante, nacida por cesárea por alteración de flujo sanguíneo cerebral a las 37 semanas de edad gestacional, pequeña para la edad gestacional, de madre primigesta de 19 años de edad

Médico Pediatra Neonatóloga, Profesor de la Facultad de Medicina Humana de la Universidad Nacional Federico Villareal. Directora Ejecutiva de Docencia, Investigación y Atención en Neonatología en el Instituto Nacional Materno Perinatal (INMP).

Médico Residente de primer año de Neonatologia del INMP por Universidad San Martín de Porres (USMP).

Médico Residente de primer año de Neonatologia del INMP por Universidad San Martín de Porres (USMP).

Médico Residente de primer año de Neonatologia del INMP por Universidad Nacional Federico Villareal (UNFV)

Citar como: Dávila CR, Del castillo SB, Guillén R, Angulo L. Caracteristicas fenotipicas de paciente con cromosoma 7 en anillo. Rev Peru Investig Mater Perinat. 2019;8(4): 62-6

DOI https://doi.org/10.33421/inmp.2019177

Recibido: 10-10-2019 Aceptado: 12-12-2019 
con 08 controles prenatales, sin antecedentes familiares de infecciones o enfermedades genéticas. Antecedentes maternos: hemoglobina12 gr/dL, grupo sanguíneo "O" Factor Rh positivo, serología negativa para HIV y sífilis, ecografía realizada el día del parto: gestación única activa de 37 semanas, RCIU, oligohidramnios y redistribución de flujos. La niña nació con peso de $1852 \mathrm{~g}$, talla de 41,5 $\mathrm{cm}$, perímetro cefálico de $29,8 \mathrm{~cm}$ y perímetro torácico de $27 \mathrm{~cm}$. La valoración de APGAR normal. Frecuencia cardiaca 120 latidos por minuto y dificultad respiratoria leve, saturación de oxigeno en rango aceptable, con valor de hemoglobina 18,8 g/dl, grupo sanguíneo "O" factor $\mathrm{RH}$ positivo, recuento de reticulocitos 6,1 y reactantes de fase aguda normales.

Durante el examen físico se observó acrocianosis, abombamiento frontal, esclera azulada, implantación posterior de orejas, en forma de taza y pequeñas; fascies dismórfica triangular, micrognatia, pestañas largas, clinodactilia, genitales femeninos con labios mayores que no cubren labios menores, hipercromía en tercio superior de labio mayor derecho. Cursó con taquipnea transitoria del recién nacido que resolvió a las 24 horas e hipoglicemia. Durante su estancia desarrolló ictericia que recibió fototerapia durante 02 días. Dificultad para succión y deglución por lo cual recibió fisioterapia orofacial. Presentó disminución de peso $>10 \%$ en 7 días.

La evaluación neurológica reportó perímetro cefálico de $29,8 \mathrm{~cm}$ (< p3), cabalgamiento de suturas, fontanela anterior casi no palpable, movimientos espontáneos, tono y reflejos conservados, al igual que la evaluación de nervios craneales y sensibilidad fueron normales. Ecografía cerebral: normal. Se diagnosticó: microcefalia. El examen TORCH dió como resultado: IgG para citomegalovirus: positivo e IgG para rubeola : positivo. La niña permaneció hospitalizada por 12 días, luego del alta continuó seguimiento por un equipo multidisciplinario.

Fondo de ojo y emisiones otoacústicas normales.

A los 2 meses de edad la evaluación neurológica reporta perímetro cefálico 33.5 centímetros, menor de P3, compatible con microcefalia, hipertonía axial y apendicular, hiporreflexia simétrica.

A los 4 meses de edad manifestó crisis tónicas clónicas con supraversión ocular de un minuto de duración en repetidas ocasiones, tono conservado, normorreflexia y desarrollo psicomotor normal, inició tratamiento con carbamazepina por diagnóstico de epilepsia.

El encefalograma fue anormal, epileptiforme, con puntas escasas y P-O centroparietales, patrón de sueño normal. Resonancia magnética cerebral no mostró alteraciones.

Curso con anemia (Hemoglobina: 10.2gr/dl) El perímetro cefálico, peso y talla se encontraban menor al P3. A los 6 meses inició la consejería nutricional.

La evaluación por genética, reportó microcefalia, dismorfia craneofacial, hemangioma plano en frente, asimetría de hemicuerpo derecho, manchas café con leche en labios mayores y muslo. Cariotipo en sangre periférica, técnica de bandeo GTG, en 25 metafases evaluadas se observaron 46 cromosomas, $\mathrm{XX}$, detectándose la presencia de un cromosoma 7 en anillo [r(7) (p22q36)].

Figura 1. Cariotipo 46, XX, r(7) (p22q36) [25]

(A)


La evaluación cardiológica no mostró alteraciones, e inició la evaluación y seguimiento por odontopediatría por alto riesgo de caries. La ecografia renal y ginecológica no mostró alteraciones.

Al año 3 meses la evaluación tiroidea presentó TSH: $11,07 \mathrm{ulU} / \mathrm{ml}$, T3 libre: $4,75 \mathrm{pg} / \mathrm{ml}$, T4 libre 0,94ng/dl, anticuerpo antiroideo antiperoxidasa (Anti TPO): 2,9UI/ $\mathrm{ml}$, Anticuerpo antitiroideo antitiroglobulina: 25,2Ul/ $\mathrm{ml}$, y la Gamagrafía de tiroides mostró tenue presencia de imagen y captación tardía de la glándula tiroidea en la región cervical anterior, lo cual sugiere hipofunción (hipotiroidismo) y elevada captación de trazador a nivel de la cavidad oral, compatible con tejido ectópico.

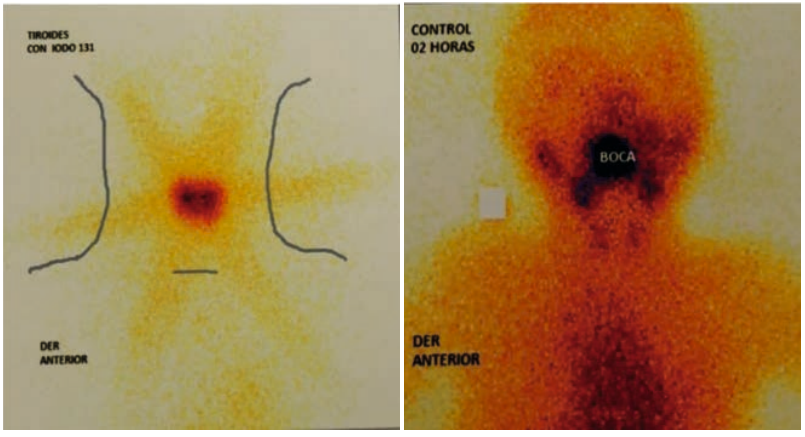

Figura 2. Gammagrafía tiroidea, muestra tiroides y captación ectópica de boca.

La evaluación hematológica mostró recuperación de la anemia, con nivel de hemoglobina $12,4 \mathrm{~g} / \mathrm{dl}$, ferritina $13,3 \mathrm{ng} / \mathrm{ml}$, vitamina B12: $277 \mathrm{pg} / \mathrm{ml}$, función hepática, renal y glucosa en rangos normales.

Al año 9 meses de edad continuó tratamiento con carbamazepina y levotiroxina; no reportó nuevas crisis convulsivas. Se observaron múltiples nevus pigmentados de aparición reciente en tronco y extremidades, hirsutismo dorsal, así como hemangiomas planos en cara y tronco; la actividad motora gruesa, fina, psicomotricidad, atención, actividades para la edad reflejan un desarrollo psicomotor normal, alteración de lenguaje y curvas de peso, talla y perímetro cefálico por debajo del P3. Figuras 3 y 4 .

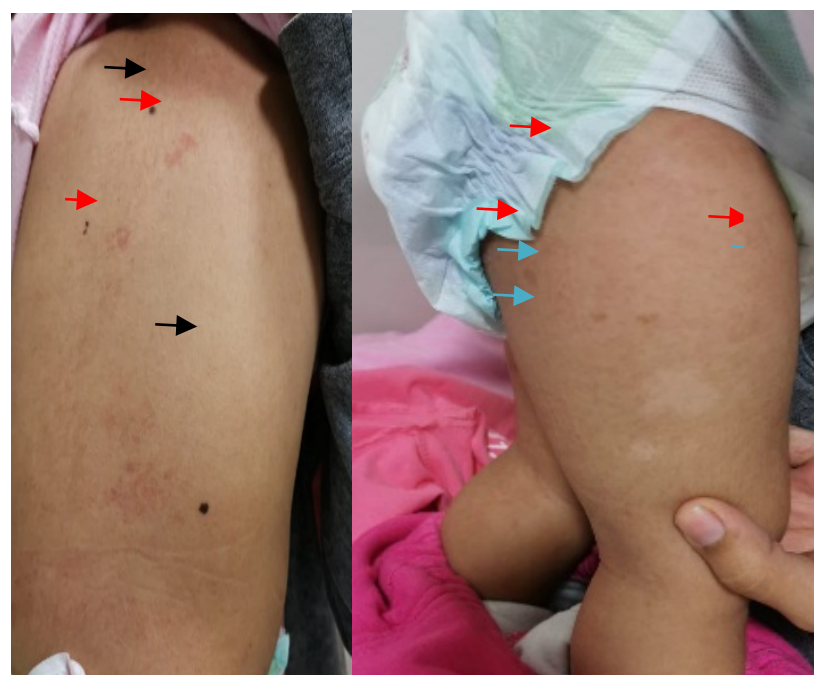

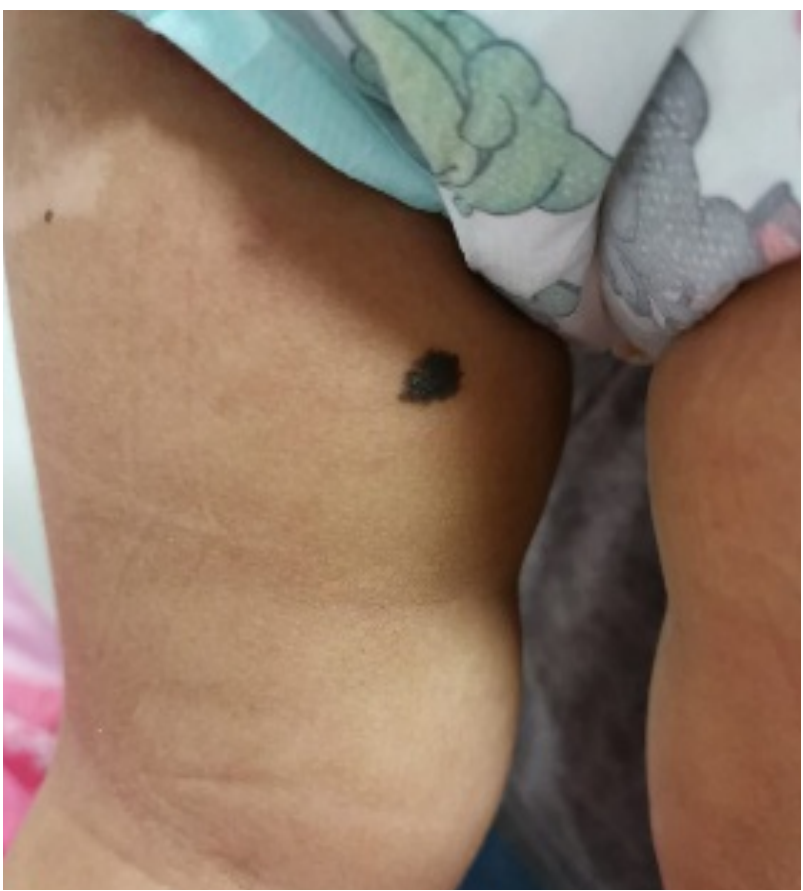

Figura 3. Manchas café con leche (flecha roja), nevus pigmentados (flecha negra) y zonas hipocrómicas (flecha azul).

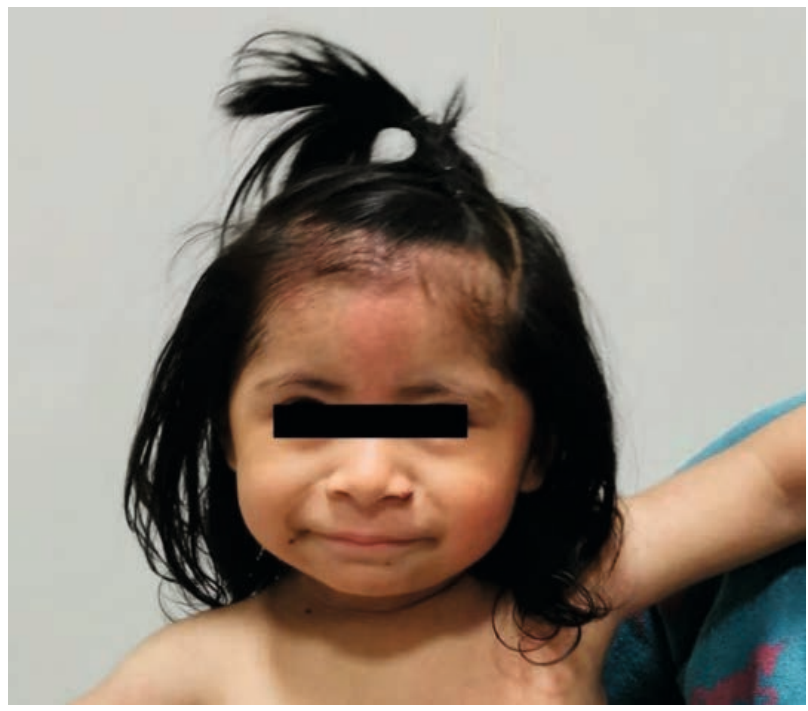

Figura 4. Paciente con cromosoma 7 en anillo.

La paciente continúa en seguimiento por equipo multidisciplinario en nuestra institución.

\section{DISCUSIÓN}

El primer caso de un paciente con Cromosoma en anillo fue reportado en 1981 por Cote y cols; independiente del cromosoma involucrado. La rotura en ambos brazos del cromosoma afectado, con fusión de los fragmentos distales da lugar a un cromosoma en anillo, refleja una alteración en la estructura del material genético. Los cromosomas anulares constitucionales ocurren en aproximadamente 1 / 50,000 fetos humanos ${ }^{2}$.

En 1973 se reportaron los primeros dos casos de cromosoma 7 en anillo. La asociación de cuadro clínico guarda relación en la posición del punto de interrupción? 
Kosztolanyi (1987) relacionó el retraso en el crecimiento severo con hallazgos de anillos grandes ${ }^{2}$.

Velagaleti, reportó la clasificación de los casos con presencia de cromosomas 7 en anillo en dos grupos: (1) los pacientes con una línea celular que tiene 47 cromosomas y el cromosoma 7 en anillo es un supernumerario, conformando una trisomia parcial. (2) presencia de línea celular con un cromosoma 7 en anillo grande, que reemplaza a uno de los cromosomas normales 7 (monosomía parcial) presentando estos últimos el cuadro similar; correspondiendo nuestra paciente al segundo grupo 8 .

Koiffmann et al en $1990^{\circ}$, reportó el caso de un varón de 39 años,con cromosoma 7 en anillo, microcefalia, fisura palpebral, cejas inclinadas hacia arriba, nariz picuda, narinas hipoplásicas, orejas altas, labios delgados, filtrum largo, hipoplasia malar, cifoscoliosis, pezones separados, nevos pigmentados, manos pequeñas con acortamiento bilateral de la segunda falange de los quintos dedos, pies pequeños con sindactilia parcial bilateral de las falanges del tercer y cuarto dedo del pie. Retraso mental severo, habla disártrica, e incontinencia urinaria nocturna, deficiencia de crecimiento y anomalías oculares y genitales.

Velagaleti (2002) reportó el caso de un niño de 12 años con un retraso significativo del desarrollo, lesiones dermatológicas con pigmentación variable, hipogenitalismo, dextroscoliosis lumbar, anomalías cerebelosa y oftalmológicas; nevus melanocítico y dificultad del habla ${ }^{8}$.

Tsai (2011) reportó un caso de una lactante de año 10 meses, con cromosoma 7 en anillo supernumerario con retraso de crecimiento, microcefalia, hipotelorismo, hipoplasia de línea media facial, estenosis coanal, arco del paladar elevado, retraso del habla y sindactilia subcutánea de los tercer y cuarto dedo del pie $^{3}$.

Kaur et al reportaron el caso de una paciente adulta procedente de India, con fascies dismórfica, fisuras papales vertebrales hacia arriba, nariz ancha, narinas estrechas, labios grandes, cuello corto, pezones muy separados, talla corta, microcefalia, manos y pies grandes, nevos pigmentados oscuros sobre el abdomen, el tórax y el muslo, retraso de lenguaje y deficiencia intelectual ${ }^{2}$. Shetty et al (2006), Hamdi (2014) y Jain at el (2018) reportaron la asociación de pacientes con cromosoma 7 en anillo y linfoma hepatoesplénico de células T (HSTL). Liu et al. (2016) reportó un caso de asociación con leucemia mieloide aguda de mal pronóstico ${ }^{10-13}$.

Henderson presentó 02 casos en asociación con holoprosencefalia (HPE), una falla del cerebro anterior para separarse completamente en dos hemisferios durante desarrollo ${ }^{14}$.

A la fecha encontramos registros de 23 pacientes reportados en el mundo, quienes comparten características tales como: curvas de crecimiento pondoestatural menor al P3,microcefalia, retraso mental, asimetría facial, hipertelorismo, fisuras palpebrales, orejas pequeñas, anomalías esqueléticas, retraso de lenguaje y nevos pigmentados y manchas café con leche ${ }^{3,15-19}$.

Las características menos frecuentes reportadas son: hoyuelo sacro, craneosinostosis, cuarto metacarpo corto, espina bífida, pectus excavatum, cifoscoliosis, proptosis, ptosis, microcórnea, coloboma coroideo, alteración de dentina dentaria, atrofia de nervio óptico, catarata, nistagmo, ptosis del párpado, testículos no descendidos, pene pequeño e hipospadias ${ }^{3,15-19}$.

Es probable que la gravedad fenotípica del cromosoma 7 en anillo está correlacionada con el grado de desequilibrio citogenético del cromosoma 7. Además algunos de los genes ubicados dentro de la región del cromosoma 7 afectada están asociados con las habilidades necesarias para el desarrollo del habla cognitivo.

Nuestra paciente actualmente de 1año 9 meses, cursa con casi todas las características más frecuentes, además de hipofunción tiroidea, no presenta compromiso de inteligencia, ni signos de retraso mental ó alteración hematológica compatible con linfoma ó leucemia.

En conclusión, aunque la prevalencia mundial del cromosoma 07 en anillo es menor a 1 en 1000000 de casos, se debe tener en cuenta ante la presencia del fenotipo reportado, en particular el déficit del crecimiento pondo estatural y retraso del habla, en el que se descarta otras comorbilidades, recomendamos la evaluación de cariotipo y función tiroidea.

La importancia de este diagnóstico radica en las múltiples asociaciones que esta patología presenta, siendo una de las más importantes y de peor pronóstico, las alteraciones onco hematológicas, observándose Leucemia Mieloide Aguda hasta en un $2 \%$ de estos pacientes.

Se trata del primer caso reportado de una paciente con Cromosoma 07 en anillo, con características fenotípicas y cariotipo compatible. Hasta el momento, la paciente no ha presentado asociaciones hemato-oncológicas, lo que compromete el grupo de investigación continuar con un seguimiento multidisciplinario exhaustivo para el manejo integral de la paciente.

\section{AGRADECIMIENTOS}

Se agradece la colaboración de los padres de la menor que autorizaron la publicación.

Financiamiento: Los autores declaran no contar con subvenciones de ningún tipo.

Conflicto de interés: Los autores declaran no tener ningún conflicto de intereses

\section{REFERENCIAS BIBLIOGRÁFICAS}

1. National Human Genome Research Institute. [Internet] Monosomía [Citado 2019 Diciembre 09]. Disponible en https://www.genome.gov/es/genetics-glossary/Monosomia

2. Kaur A, Dhillona S, Gargb P, et al. Ring chromosome 7 in an Indian woman. Journal of Intellectual and Developmental Disability, 2008, 33:1, 87-94

3. Tsai L, Lee K, Fang J, et al. Molecular cytogenetic analysis and clinical manifestations of a case with de novo mosaic ring chromosome 7. Molecular Cytogenetics 2011, 4:5, 1-5

4. Orphanet: Portal de información de enfermedades raras y medicamentos huérfanos [internet]. Comisión europea, Francia; 1997. Sindrome del cromosoma 7 en anillo. [Citado 2019 Diciembre 11]; [9 screens] Disponible en: https://www.orpha.net/consor

5. MalaCards: HUMAN DISEASE DATABASE [internet]. Weizmann Institute of Science: Gene Cards Suite; c2017. Ring Chromosome 7 [Citado 2019 Diciembre 10]; [7 screens] Disponible en: https://www.malacards.org/card/ ring_chromosome_7 
6. Inaba $\mathrm{T}$, Honda $\mathrm{H}$, Matsui $\mathrm{H}$, et al. The Enigma of Monosomy 7. American Society of Hematology 2018. April 3, 2018. Disponible en: www.bloodjournal.org

7. Zackai EH, Breg WR. Ring chromosome 7 with variable phenotypic expression. Cytogenet Cell Genet. 1973;12:408.

8. Velagaleti G, Jalal S, Kukolich M, Lockhart L, Tonk V. De novo supernumerary ring chromosome 7 : first report of a non-mosaic patient and review of the literature. Clinica Genetics, 61(3), 202-206. (2002). doi:10.1034/j.1399$0004.2002 .610306 x$

9. Koiffmann C, Diament A, De Souza D, Wajntal A. Ring chromosome 7 in a man with multiple congenital anomalies and mental retardation. Med Genet 1990; 27: 462-464

10. Shetty S, Mansoor A, Roland B. Ring chromosome 7 with amplification of $7 q$ sequences in a pediatric case of hepatosplenic T-cell lymphoma. Cancer Genetics and Cytogenetics.2006;167:161-163

11. Hamdi A, Afrough A, Muzzafar T,et al. Donor CelleDerived Myelodysplastic Syndrome with ring chromosome 7 after allogeneic hematopoietic Stem Cell Transplant in 2 patients with lymphomas as primary disease clinical lymphoma, myeloma \& leukemia. Published by Elsevier Inc. 2014;14(5): e151-5.

12. Jain H, Shetty D , Jain H, Manju Sengar M , Navin Khattry Nc , P.G. Subramanian P. A rare case of hepatosplenic үठT-cell lymphoma expressing CD19 with ring chromosome 7 and trisomy 8. Cancer Genetics.2018; 228-229:17-20.

13. Liu K, Tirado C. Ring Chromosome 7: A Rare Structural Abnormality in Acute Myeloid Leukemia (AML). J Assoc Genet Technol. 2016;42(4):181-186

14. Henderson L, Corson V, Saul D, Anderson C et al. First Trimester Diagnosis of Holoprosencephaly Secondary to a Ring Chromosome 7. Hindawi Publishing Corporation. Case Reports in Genetics. Volume 2013, Article ID 578202 , 3 pages. http://dx.doi.org/10.1155/2013/578202
15. DeLozier CD, Theintz G, Sizonenko P, Engel E. A fourth case of ring chromosome 7. Clin Genet. 1982;22:90-8.

16. Barros L, Perez A, Brunoni D, Andrade J. Relato de un caso de cromosoma 7 anular. Cienc Cult.1986;38 (suppl):849.

17. Lichtenbelt K, Hochstenbach R, Dam V, Eleveld M, Poot M, y Beeme F. Supernumerary Ring Chromosome 7 Mosaicism: Case Report, Investigation of the Gene Content, and Delineation of the Phenotype. American Journal of Medical Genetics.2005;132A:93-100.

18. Bertini V, Valetto A, Uccelli A, et al. Molecular Cytogenetic Characterization of a de novo Mosaic Supernumerary Ring Chromosome 7: Report of a New Patient. American Journal of Medical Genetics. 2008;146 A: 2955-2959.

19. Salas-Labadía C, Cervantes-Barragán D, Cruz-Alcívar R, et al. Cytogenomic and phenotypic analysis in low-level monosomy 7 mosaicism with non-supernumerary ring chromosome 7. Am J Med Genet Part A. 2014;164A:1765-1769.

\section{Correspondencia}

Carmen Rosa Dávila Aliaga,

Dirección: Jr. Santa Rosa \# 941 - Cercado de Lima

Correo: davilacarmen@hotmail.com

Teléfono 3310209 - 999042084. 